\title{
Effect of alvimopan on gastrointestinal recovery and length of hospital stay after retroperitoneal lymph node dissection for testicular cancer
}

\author{
Kushan D Radadia ${ }^{1,2}$, Nicholas J Farber ${ }^{1,2}$, Alexandra L Tabakin ${ }^{1,2}$, Wei Wang ${ }^{3}$, Hiren V \\ Patel $^{1,2}$, Charles F Polotti ${ }^{1,2}$, Robert E Weiss ${ }^{1}$, Sammy E Elsamra ${ }^{1,2}$, Isaac Y Kim ${ }^{1,2}$, Eric A \\ Singer ${ }^{1,2}$, Mark N Stein ${ }^{4}$, Tina M Mayer ${ }^{4}$, and Thomas L Jang ${ }^{1,2}$ \\ ${ }^{1}$ Section of Urologic Oncology, Rutgers Cancer Institute of New Jersey, New Brunswick, USA \\ ${ }^{2}$ Division of Urology, Department of Surgery, Rutgers Robert Wood Johnson Medical School, New \\ Brunswick, USA \\ ${ }^{3}$ Department of Biostatistics, Rutgers-School of Public Health, Piscataway, USA \\ ${ }^{4}$ Division of Genitourinary Medical Oncology, Department of Medicine, Rutgers Cancer Institute of \\ New Jersey and Rutgers Robert Wood Johnson Medical School, New Brunswick, USA
}

\section{Abstract}

Objective: Alvimopan use has reduced the length of hospital stay in patients undergoing major abdominal surgeries and radical cystectomy. Retroperitoneal lymph node dissection for testicular cancer may be associated with delayed gastrointestinal recovery prolonging hospital length of stay. We evaluate whether alvimopan is associated with enhanced gastrointestinal recovery and shorter hospital length of stay in men undergoing retroperitoneal lymph node dissection for testicular cancer.

Materials and methods: From 2010 to 2016, 29 patients underwent open, transperitoneal bilateral template retroperitoneal lymph node dissection. Data for patients who received alvimopan were prospectively collected and compared to a historical cohort of patients who did not receive alvimopan. Primary outcome measures were length of stay and recovery of gastrointestinal

\footnotetext{
Reprints and permissions: sagepub.co.uk/journalsPermissions.nav

Corresponding author: Thomas L Jang, Division of Urology, Urologic Oncology Program, Rutgers Cancer Institute of New Jersey, 195 Little Albany Street, New Brunswick, NJ 08901, USA. jangtl@ cinj.rutgers.edu.

Contributorship: KR, NF and TJ contributed to the study design. KR, NF, AT, WW and HP contributed to data collection and analysis. KR, NF, AT, HP and TJ contributed to drafting the manuscript. All authors reviewed and edited the manuscript and approved the final version of the manuscript.

Conflicting interests

The authors declare that there is no conflict of interest.

Ethical approval

Ethical approval for this study was obtained from Rutgers University Biomedical and Health Sciences institutional review board (Pro20160000812).

Informed consent

Not applicable. Informed consent was not sought for the present study because this was a retrospective study that used data with no personal identifiers.

Guarantor

TJ
} 
function. Descriptive statistics were reported. Time-to-event outcomes were evaluated using cumulative incidence curves and log rank test. Factors associated with length of stay were analyzed for correlation using multiple linear regression.

Results: Of 29 men undergoing retroperitoneal lymph node dissection, eight received alvimopan and 21 did not. The two cohorts were well matched, with no significant differences. In the alvimopan cohort compared with those who did not receive alvimopan median time to return of flatus was 2 versus 4 days ( $p=0.0002$ ), and median time to first bowel movement was 2.5 versus 5 days ( $p=0.046$ ), respectively. Median length of stay in the alvimopan cohort was 4 days versus 6 days in those who did not receive alvimopan $(p=0.074)$. In adjusted analyses, receipt of alvimopan did not influence length of stay.

Conclusion: Alvimopan may facilitate gastrointestinal recovery after retroperitoneal lymph node dissection for testicular cancer. Whether this translates into reduced length of stay needs to be determined by randomized controlled trials using larger cohorts.

Level of evidence: $3 b$.

\section{Keywords}

Alvimopan; gastrointestinal recovery; length of hospital stay; retroperitoneal lymph node dissection; testicular cancer

\section{Introduction}

Testicular cancer or germ cell tumor (GCT) is the most common solid tumor in men between the ages of 20 and 34 years. ${ }^{1}$ With current multidisciplinary approaches to treatment, longterm survival rates, even for men with metastatic GCT, exceed $90 \%$. This is largely due to the development of cisplatin-based chemotherapy, reliable tumor markers available for this disease, and the appropriate integration of and refinements in surgery over time. ${ }^{2-4}$ Given the high cure rates in these men, treatment objectives should focus on minimizing treatmentrelated toxicities.

Retroperitoneal lymph node dissection (RPLND) is a well-established therapy to treat GCTs in the primary or post-chemotherapy setting. RPLND can be technically challenging, with overall complication rates for primary RPLND and post-chemotherapy RPLND reported to be $10-24 \%{ }^{5-8}$ and $20-30 \%,{ }^{9}, 10$ respectively. Postoperative paralytic ileus, which can delay immediate recovery, increase postoperative morbidity, extend a patient's hospital length of stay (LOS), and increase costs, ${ }^{11,12}$ has been reported in up to $18 \%$ of men undergoing primary RPLND and up to $21 \%$ of men undergoing post-chemotherapy RPLND. ${ }^{7}$ To facilitate quicker return of bowel function and shorten LOS, researchers have reported on an extraperitoneal approach to RPLND. ${ }^{13}$

Alvimopan (trade name Entereg; Merck) is an oral medication that works as a $\mu$-opioid receptor antagonist to reduce the incidence and severity of postoperative ileus in postsurgical patients. Randomized controlled trials in the general surgical and urological literature have demonstrated the benefit of alvimopan in reducing LOS and decreasing the incidence of postoperative ileus. ${ }^{14,15}$ In the urological field, alvimopan has been studied in 
patients undergoing radical cystectomy with urinary diversion for invasive bladder cancer. ${ }^{16}$ In fact, enhanced recovery after surgery (ERAS) protocols, which are evidence-based pathways that aim to optimize and standardize the perioperative care of patients receiving complex surgeries, have adopted alvimopan as a standard part of the ERAS protocol, to shorten LOS without compromising post-surgery outcomes. ${ }^{17}$

To our knowledge, alvimopan has yet to be investigated in the testicular cancer population after surgery. In this study, we evaluate whether alvimopan is associated with enhanced gastrointestinal recovery and shorter hospital LOS in men undergoing RPLND for testicular cancer.

\section{Materials and methods}

\section{Study population}

Men who received a RPLND for testicular cancer from March 2010 to August 2016 at our center were included in this study. All patients underwent an open, transperitoneal bilateraltemplate RPLND (nerve sparing when feasible) by a single surgeon. Patients who, for whatever reason, received prescription opioid narcotic medications prior to RPLND were excluded from the study. The study was reviewed and approved by the institutional review board at the Rutgers Cancer Institute of New Jersey.

\section{Description of the intervention}

Patients received a single dose of alvimopan $12 \mathrm{mg}$ tablet orally 30 minutes preoperatively and then alvimopan was readministered every 12 hours postoperatively for up to 14 doses and discontinued only on discharge or if the patient received the maximum 15 doses. ${ }^{18}$

\section{Post-surgical care}

All patients received general anesthesia. Post-surgery pain management was provided by intravenous hydromorphone patient controlled analgesia until advanced to and tolerating a clear liquid diet, after which they were placed on either oral hydromorphone or acetaminophen/oxycodone.

Diet and activity were advanced as tolerated. On the first postoperative day, patients were started on sips of clear liquids if it was tolerated and the diet was sequentially advanced during hospitalization to clear liquid diet and then regular diet when tolerated. Patients were considered to be tolerating the diet if they did not experience nausea, vomiting, or abdominal distension.

Patients were seen twice daily by the operative team, between 6 a.m. and 8 a.m. and between 5 p.m. and 7 p.m. and questioned during each visit whether the passage of flatus and/or first bowel movement has occurred.

Patients were deemed appropriate for discharge when no complications were present, they were tolerating oral nutrition, pain was controlled on oral analgesics, and gastrointestinal function had returned (flatus or bowel movement). 


\section{Study endpoints}

Two comparative arms, those who received alvimopan during their RPLND hospitalization and those who did not, were assessed. Data for men who received alvimopan were prospectively collected and compared to a historical cohort of patients who did not receive alvimopan. Primary outcomes measured were length of hospital stay and recovery of gastrointestinal function, which we defined as time to return of flatus and time to first bowel movement.

\section{Statistical analysis}

Descriptive statistics were calculated for patient clinical and demographic characteristics, according to whether patients received alvimopan or not. Univariate comparison for each variable was performed using chi-square tests for categorical variables and Mann-WhitneyWilcoxon tests for continuous variables. For each of the time to event outcomes, namely time to return of flatus and time to first bowel movement after surgery, cumulative incidence curves were plotted. The log rank test was used to determine the significance of treatment effects between the two arms. Potential factors associated with hospital LOS were analyzed for correlation using a multiple linear regression approach. Both unadjusted and adjusted regression analyses were performed. Time-to-event analyses and multiple linear regression were conducted using R statistical software version 3.4.2 (https://www.r-project.org/). Statistical tests were determined to be significant at a two-sided $p$-value of less than 0.05 .

\section{Results}

From March 2010 to August 2016, 29 patients who underwent RPLND by a single surgeon met the inclusion criteria. Of those patients, 23 (79.3\%) received RPLND in the postchemotherapy setting while six (20.7\%) received RPLND in the primary setting. When comparing the cohort of patients who received alvimopan to those who did not, there were no significant differences between the two groups with respect to age, proportion with nonseminomatous GCT histology, proportion who received post-chemotherapy RPLND, preoperative mass size, body mass index, smoking history, comorbidity index score, estimated blood loss, or the need for transfusion (Table 1).

In total, six patients (20.1\%) had complications at 90 days, of which one (3.4\%) was Clavien grade 1 (nausea requiring anti-emetics) and two (6.8\%) were Clavien grade 2 (one patient developed a pulmonary embolus requiring anticoagulation, another developed Clostridium difficile infection requiring antimicrobial treatment). There were two Clavien grade $3 \mathrm{a}$ complications (6.8\%), one of which was a symptomatic lymphocele requiring image-guided drainage and another developed biliary obstruction secondary to pre-existing choledocholithiasis (unrelated to RPLND) requiring endoscopic retrograde cholangiopancreatography. Finally, one patient (3.4\%) experienced a Clavien grade 4 complication, namely post-surgery acute respiratory distress syndrome from bleomycininduced pneumonitis requiring intensive care monitoring and temporary ventilatory support. Table 2 reports Clavien-Dindo complications at 90 days, according to whether alvimopan was received or not. 
Alvimopan use did appear to enhance gastrointestinal recovery, decreasing both the median time to flatus and the median time to first bowel movement after surgery. More specifically, the median time to the return of flatus in men receiving alvimopan was 2 days compared to 4 days for those who did not (log rank test $p$-value 0.0002) (Figure 1(a)). The median time to first bowel movement in men receiving alvimopan was 2.5 days versus 5 days for those who did not (log rank test $p$-value 0.046) (Figure 1(b)).

Median hospital LOS for men receiving alvimopan compared with those men who did not was 4 days versus 6 days, respectively ( $p=0.074$ ). To explore the association of several factors that may increase LOS after RPLND, we analyzed, using a multiple linear regression approach, whether receipt of alvimopan (vs. no receipt), RPLND approach (primary vs. post-chemotherapy) and Clavien grade complications, were associated with LOS. Both unadjusted and adjusted regression analyses demonstrate that LOS is influenced mainly by Clavien grade complications, and was not associated with alvimopan use or with the setting in which RPLND was performed (primary vs. post-chemotherapy) (Table 3). For example, on adjusted analyses, there was an incremental increase in hospital LOS (days) according to Clavien grade classification, with patients who had Clavien grade 1, 2 and 3a complications staying 1.9, 3.1 and 4.1 days longer, respectively, than those without complications (Clavien grade 0$),(p=0.10, p=0.0006, p=0.0002$, respectively $)$.

\section{Discussion}

Major abdominal surgeries increase the risk of postoperative paralytic ileus secondary to bowel manipulation, spinal-intestinal neural reflexes, secretion of inflammatory mediators, anesthetics, and postoperative opioid use. ERAS protocols are standardized, multi-modal perioperative care pathways developed to reduce morbidity after major abdominal surgery. Alvimopan has been incorporated into these ERAS protocols and has been shown to improve outcomes in the general surgical literature. ${ }^{14,19}$ This success has prompted investigations of its use in other types of surgeries, in particular urological procedures. Two randomized controlled trials demonstrated that alvimopan accelerates gastrointestinal recovery in patients who undergo radical cystectomy with urinary diversion. ${ }^{16,20}$ Lee et al. showed that alvimopan accelerated gastrointestinal recovery, defined as tolerating a diet and first bowel movement, compared to placebo, in patients undergoing radical cystectomy with urinary diversion. In their study, investigators showed that the alvimopan cohort experienced a quicker time to first bowel movement ( 5.5 days vs. 6.8 days, $p<0.0001)$ and shorter mean LOS (7.4 vs. 10.1 days, $p=0.0051) .{ }^{16}$ Likewise, similar findings were demonstrated in a study by Kauf et al., which reported a mean LOS reduction of 2.63 days with the use of alvimopan $(p=0.005){ }^{20}$

Theoretically, alvimopan may accelerate gastrointestinal recovery and shorten LOS after RPLND, a urological surgery with reported ileus rates of up to $20 \%$, as it requires exteriorization of bowel to expose the great vessels. ${ }^{7}$ To our knowledge, the use of alvimopan to enhance post-surgical recovery after RPLND for men with testicular cancer has yet to be reported. 
Our analysis aimed to determine whether alvimopan enhanced gastrointestinal recovery and shortened hospital LOS in this population. Several key findings were observed. First, alvimopan use did appear to facilitate gastrointestinal recovery, significantly decreasing both the median time to flatus and the median time to first bowel movement after surgery. Return of flatus and of bowel movements occurred 2 and 2.5 days earlier, respectively, in the cohort of men who received alvimopan when compared to those who did not. However, in our study cohort, enhanced gastrointestinal recovery did not translate into shorter LOS for men receiving alvimopan. Although there was a trend towards shorter median hospital LOS for the alvimopan cohort ( 4 days vs. 6 days, $p=0.074$ ), these results were not statistically significant, nor was alvimopan use, on unadjusted and adjusted analyses, associated with hospital LOS. To further determine the relative influence of co-dependent factors (i.e. alvimopan use, Clavien complications, setting of RPLND) that may influence LOS and assess each factor's relative influence, we performed a multiple linear regression analysis. On unadjusted and adjusted analysis, the major determinant influencing LOS was the presence of complications postoperatively. Not surprisingly, there was an incremental increase in hospital LOS (days) according to Clavien grade classification, with patients who had higher grade complications having extended LOSs. Receipt of alvimopan or whether patients received a RPLND in the primary or post-chemotherapy setting did not influence hospital LOS.

Our findings are analogous with the published literature demonstrating that alvimopan enhances gastrointestinal recovery after colorectal surgery and radical cystectomy. ${ }^{14,16,20-26}$ However, we were not able to demonstrate a reduction in LOS with alvimopan use.

This study has several limitations. First, our pilot study comprised a small sample size of men from a single institution. Second, although the alvimopan cohort was prospectively evaluated, the comparative arm included a historical cohort in which data collection was retrospective in nature. Despite this, our cohorts were well matched in terms of clinical and demographic characteristics, with no significant differences in the variables analyzed. Finally, hospital LOS is a complex endpoint to evaluate, as many factors, some codependent, in the pre, intra and postoperative setting, can influence its outcome. Although we found a trend towards shorter LOS in the alvimopan cohort (4 days vs. 6 days), the results were not statistically significant and when subjected to a multivariable regression analysis, alvimopan use did not appear to influence LOS in this population.

Our initial exploratory study, although limited by its small sample size, identifies a potentially promising modality to be incorporated into ERAS protocols to enhance gastrointestinal recovery in patients with testicular cancer undergoing RPLND. Randomized controlled trials with larger cohorts will be needed to validate the findings of enhanced gastrointestinal recovery in this population and to clarify whether the receipt of alvimopan can reduce LOS and hospitalization costs.

The use of alvimopan may facilitate gastrointestinal recovery in men undergoing RPLND for testicular cancer, reducing the time to flatus and reducing the time to first bowel movement after surgery. Whether this translates into reduced hospital LOS and decreased 
hospitalization costs needs to be determined by randomized controlled trials using larger cohorts.

\section{Conclusion}

The use of alvimopan may facilitate gastrointestinal recovery in men undergoing RPLND for testicular cancer, reducing the time to flatus and reducing the time to first bowel movement after surgery. Whether this translates into reduced hospital LOS and decreased hospitalization costs needs to be determined by randomized controlled trials using larger cohorts.

\section{Acknowledgements}

Funding

This work is supported by a grant from the National Cancer Institute P30 CA-072720 (Rutgers Cancer Institute of New Jersey Biometrics Shared Resource).

\section{References}

1. Siegel RL, Miller KD, Fedewa SA, et al. Colorectal cancer statistics, 2017. CA Cancer J Clin 2017; 67: 177-193. [PubMed: 28248415]

2. Einhorn LH. Treatment of testicular cancer: a new and improved model. J Clin Oncol 1990; 8: 1777-1781. [PubMed: 1700077]

3. Carver BS, Serio AM, Bajorin D, et al. Improved clinical outcome in recent years for men with metastatic nonseminomatous germ cell tumors. J Clin Oncol 2007; 25: 5603-5608. [PubMed: 17998544]

4. National Cancer Institute. SEER Stat fact sheets: testis cancer https://seer.cancer.gov/statfacts/html/ testis.html (accessed 30 June 2018).

5. Baniel J, Foster RS, Rowland RG, et al. Complications of primary retroperitoneal lymph node dissection. J Urol 1994; 152 (2 Pt 1): 424-427. [PubMed: 8015086]

6. Heidenreich A, Albers P, Hartmann M, et al. Complications of primary nerve sparing retroperitoneal lymph node dissection for clinical stage I nonseminomatous germ cell tumors of the testis: experience of the German Testicular Cancer Study Group. J Urol 2003; 169: 1710-1714. [PubMed: 12686815]

7. Subramanian VS, Nguyen CT, Stephenson AJ, et al. Complications of open primary and postchemotherapy retroperitoneal lymph node dissection for testicular cancer. Urol Oncol 2010; 28: 504-509. [PubMed: 19097812]

8. Stephenson AJ and Sheinfeld J. The role of retroperitoneal lymph node dissection in the management of testicular cancer. Urol Oncol 2004; 22: 225-233; discussion 234-225. [PubMed: 15271322]

9. Baniel J, Foster RS, Rowland RG, et al. Complications of post-chemotherapy retroperitoneal lymph node dissection. J Urol 1995; 153 (3 Pt 2): 976-980. [PubMed: 7853586]

10. Heidenreich A, Thuer D and Polyakov S. Postchemotherapy retroperitoneal lymph node dissection in advanced germ cell tumours of the testis. Eur Urol 2008; 53: 260-272. [PubMed: 18045770]

11. Delaney CP, Senagore AJ, Viscusi ER, et al. Postoperative upper and lower gastrointestinal recovery and gastrointestinal morbidity in patients undergoing bowel resection: pooled analysis of placebo data from 3 randomized controlled trials. Am J Surg 2006; 191: 315-319. [PubMed: 16490538]

12. Baig MK and Wexner SD. Postoperative ileus: a review. Dis Colon Rectum 2004; 47: 516-526. [PubMed: 14978625] 
13. Syan-Bhanvadia S, Bazargani ST, Clifford TG, et al. Midline extraperitoneal approach to retroperitoneal lymph node dissection in testicular cancer: minimizing surgical morbidity. Eur Urol 2017; 72: 814-820. [PubMed: 28325537]

14. McNicol ED, Boyce D, Schumann R, et al. Mu-opioid antagonists for opioid-induced bowel dysfunction. Cochrane Database Syst Rev 2008; 2: CD006332.

15. Sultan S, Coles B and Dahm P. Alvimopan for recovery of bowel function after radical cystectomy. Cochrane Database Syst Rev 2017; 5: CD012111.

16. Lee CT, Chang SS, Kamat AM, et al. Alvimopan accelerates gastrointestinal recovery after radical cystectomy: a multicenter randomized placebo-controlled trial. Eur Urol 2014; 66: 265-272. [PubMed: 24630419]

17. Daneshmand S, Ahmadi H, Schuckman AK, et al. Enhanced recovery protocol after radical cystectomy for bladder cancer. J Urol 2014; 192: 50-55. [PubMed: 24518775]

18. US Food and Drug Administration (FDA). Alvimopan prescribing information 2008 www.accessdata.fda.gov/drugsatfda_docs/label/2008/0217751bl.pdf (accessed 30 June 2018).

19. Taguchi A, Sharma N, Saleem RM, et al. Selective postoperative inhibition of gastrointestinal opioid receptors. N Engl J Med 2001; 345: 935-940. [PubMed: 11575284]

20. Kauf TL, Svatek RS, Amiel G, et al. Alvimopan, a peripherally acting mu-opioid receptor antagonist, is associated with reduced costs after radical cystectomy: economic analysis of a phase 4 randomized, controlled trial. J Urol 2014; 191: 1721-1727. [PubMed: 24342144]

21. Viscusi ER, Goldstein S, Witkowski T, et al. Alvimopan, a peripherally acting mu-opioid receptor antagonist, compared with placebo in postoperative ileus after major abdominal surgery: results of a randomized, double-blind, controlled study. Surg Endosc 2006; 20: 64-70. [PubMed: 16333556]

22. Wolff BG, Michelassi F, Gerkin TM, et al. Alvimopan, a novel, peripherally acting mu opioid antagonist: results of a multicenter, randomized, double-blind, placebo-controlled, phase III trial of major abdominal surgery and postoperative ileus. Ann Surg 2004; 240: 728-734; discussion 734725. [PubMed: 15383800]

23. Kraft M, Maclaren R, Du W, et al. Alvimopan (entereg) for the management of postoperative ileus in patients undergoing bowel resection. P T 2010; 35: 44-49. [PubMed: 20182561]

24. Hamilton Z, Parker W, Griffin J, et al. Alvimopan in an enhanced recovery program following radical cystectomy. Bladder Cancer 2015; 1: 137-142. [PubMed: 27398398]

25. Ludwig K, Viscusi ER, Wolff BG, et al. Alvimopan for the management of postoperative ileus after bowel resection: characterization of clinical benefit by pooled responder analysis. World J Surg 2010; 34: 2185-2190. [PubMed: 20526599]

26. Manger JP, Nelson M, Blanchard S, et al. Alvimopan: a cost-effective tool to decrease cystectomy length of stay. Cent Eur J Urol 2014; 67: 335-341. 


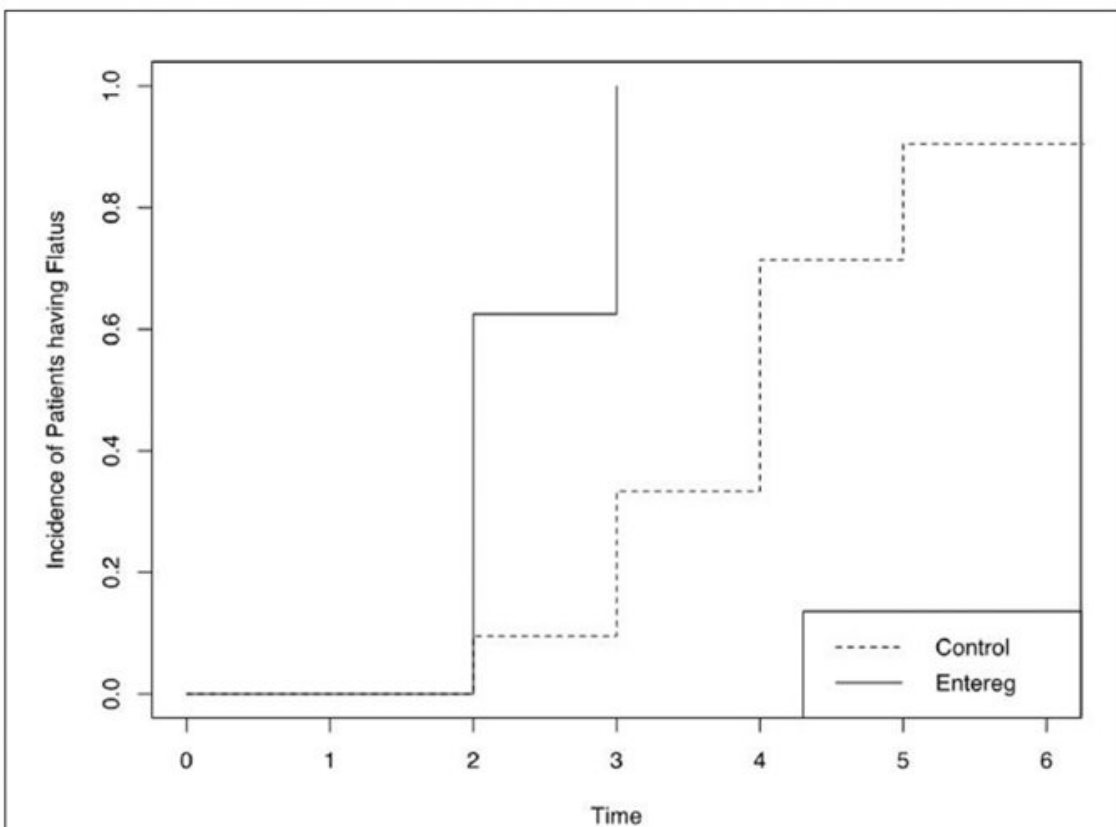

(a)

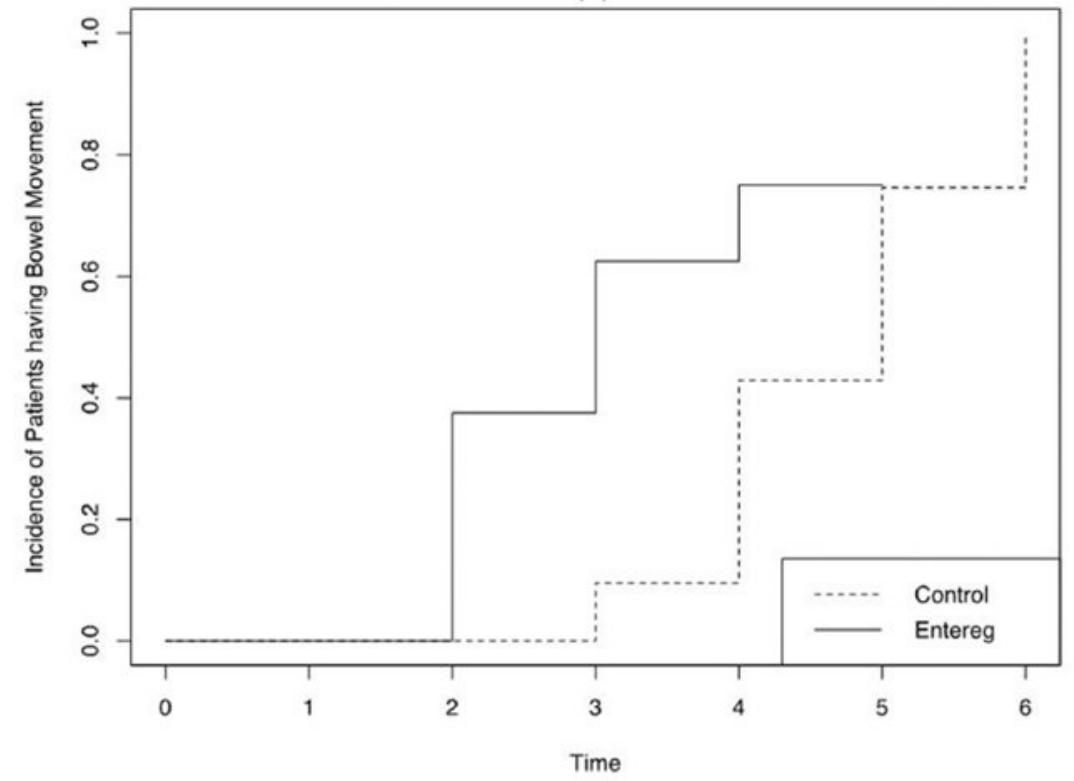

(b)

Figure 1.

(a) Time to flatus after retroperitoneal lymph node dissection (days). (b) Time to first bowel movement after retroperitoneal lymph node dissection (days). 
Table 1.

Clinical characteristics between RPLND patients who received alvimopan versus those who did not.

\begin{tabular}{|llll|}
\hline Clinical characteristics & No Alvimopan $(\boldsymbol{n = 2 1})$ & Alvimopan $(\boldsymbol{n}=\mathbf{8})$ & $\boldsymbol{p}$-value \\
\hline Median age $($ years $)$ & 27 & 29.5 & 0.783 \\
\hline No. NSGCT $(\%)$ & $20(95.2)$ & $6(75.0)$ & 0.176 \\
\hline No. post-chemotherapy $(\%)$ & $16(76.2)$ & $7(87.5)$ & 0.647 \\
\hline Mean preoperative mass size $(\mathrm{cm})$ & 2.9 & 2.61 & 0.813 \\
\hline Body mass index $\left(\mathrm{kg} / \mathrm{m}^{2}\right)$ & 32.1 & 26.8 & 0.105 \\
\hline Smoking history $(\%)$ & 38 & 37.5 & 0.659 \\
\hline Mean Charlson comorbidity index & 2.76 & 3 & 0.54 \\
\hline Mean transfusion units $(\mathrm{pRBCs})$ & 0.05 & 0 & 0.329 \\
\hline Mean EBL $(\mathrm{mL})$ & 371.1 & 493.7 & 0.477 \\
\hline
\end{tabular}

RPLND: retroperitoneal lymph node dissection; NSGCT: non-seminomatous germ cell tumor; pRBCs: packed red blood cells; EBL: estimated blood loss. 
Table 2.

Clavien-Dindo complications at 90 days.

\begin{tabular}{|c|c|c|c|}
\hline \multirow[t]{2}{*}{ Clavien grade } & \multirow{2}{*}{$\frac{\text { No alvimopan }(n=21)}{n, \% \text { of total patients }}$} & \multirow{2}{*}{$\frac{\text { Alvimopan }(n=8)}{n, \% \text { of total patients }}$} & \multirow[t]{2}{*}{ Description } \\
\hline & & & \\
\hline I & $1(3.4 \%)$ & 0 & Nausea requiring anti-emetics \\
\hline II & $1(3.4 \%)$ & $1(3.4 \%)$ & Clostridium difficile infection; pulmonary embolus \\
\hline IIIa & $2(6.9 \%)$ & 0 & $\begin{array}{l}\text { Lymphocele requiring drain; biliary obstruction secondary to pre-existing } \\
\text { choledocholithiasis requiring ERCP }\end{array}$ \\
\hline IIIb & 0 & 0 & \\
\hline IV & $1(3.4 \%)$ & 0 & ARDS secondary to bleomycin pneumonitis requiring ICU management \\
\hline $\mathrm{V}$ & 0 & 0 & \\
\hline Total & $5(17.2 \%)$ & $1(3.4 \%)$ & \\
\hline
\end{tabular}

ERCP: endoscopic retrograde cholangiopancreatography; ARDS: acute respiratory distress syndrome; ICU: intensive care unit. 
Table 3.

Multivariable analysis for factors associated with length of hospital stay.

\begin{tabular}{|c|c|c|c|c|c|}
\hline \multirow[t]{2}{*}{ Variable } & \multirow[t]{2}{*}{ Description } & \multicolumn{2}{|l|}{ Unadjusted } & \multicolumn{2}{|l|}{ Adjusted } \\
\hline & & $\begin{array}{l}\text { No. days (LOS) more or less } \\
\text { than reference }\end{array}$ & $p$-value & $\begin{array}{l}\text { No. days }(\mathrm{LOS}) \text { more or less } \\
\text { than reference }\end{array}$ & $p$-value \\
\hline \multirow[t]{2}{*}{ Alvimopan use } & No alvimopan (reference) & 0 & & 0 & \\
\hline & Alvimopan received & -2.24 & 0.36 & -0.55 & 0.24 \\
\hline \multirow[t]{2}{*}{ Setting of RPLND } & Primary (reference) & 0 & & 0 & \\
\hline & Post-chemotherapy & -0.058 & 0.98 & -0.27 & 0.65 \\
\hline \multirow[t]{5}{*}{ Clavien-Dindo grade } & 0 (reference) & 0 & & 0 & \\
\hline & 1 & 2 & 0.075 & 1.88 & 0.10 \\
\hline & 2 & 3 & 0.0007 & 3.15 & 0.0006 \\
\hline & $3 a$ & 4.5 & $<0.0001$ & 4.11 & 0.0002 \\
\hline & 4 & 30 & $<0.0001$ & 29.88 & $<0.0001$ \\
\hline
\end{tabular}

LOS: length of stay; RPLND: retroperitoneal lymph node dissection. 